\title{
Nasal glial heterotopia
}

INSERM

\section{Source}

INSERM. (1999). Orphanet: an online rare disease and orphan drug data base. Nasal glial heterotopia. ORPHA:141112

Nasal glial heterotopia is a rare developmental abnormality presenting usually at birth or in early childhood (rarely in adulthood) as a benign, non-pulsatile mass that can lead to nasal obstruction, deformation of the septum and nasal bone, and respiratory distress if untreated. Nasal glial heterotopias have no communication with the central nervous system; however an associated defect in the cribriform plate is sometimes reported. 